\title{
LES DÉFIS DE LA TRANSITION NUMÉRIQUE DANS UN PETIT MARCHÉ : LE CAS DE LA PRESSE FRANCOPHONE QUÉBÉCOISE.
}

\author{
Renaud Carbasse ${ }^{1}$
}

L'accélération de la transition numérique dans le secteur de la presse se pose de manière spécifique pour les marchés de petite taille dans lesquels le bassin de lecteurs et d'annonceurs ne permet pas de compenser efficacement les pertes de revenus observées pour les plateformes traditionnelles, ni de garantir une viabilité des activités numériques. En nous basant sur les données disponibles, nous faisons le point sur les spécificités du marché de la presse écrite quotidienne au Québec. Chaque groupe cherche à capitaliser en ligne sur des savoir-faire, modèles d'affaires et des positionnements éditoriaux antérieurs dans une situation où le déclin du lectorat survient plus tardivement et à un rythme plus lent qu'ailleurs en Amérique du nord. Les enjeux posés par la valorisation de l'offre d'information nous amènent à envisager les implications pour la production de contenus originaux francophones à l'échelle de la province.

1 Renaud Carbasse est professeur assistant au Département d'information et de communication, Université Laval (Québec, QC, Canada) et chercheur associé au Centre de recherche interdisciplinaire sur la communication, l'information et la société (CRICIS). Il est doctorant à la Faculté de Communication, Université du Québec à Montréal (Montréal, QC, Canada).

Recherches en communication, $n^{\circ} 44$ - Article publié le 23/11/2017 
Dans la myriade de restructurations socioéconomiques, cultures, politiques et sociales, favorisées par le numérique, se fait sentir de manière toujours plus pressante la question du renouvellement des modèles d'affaires de la presse écrite en ligne. Le secteur de la presse écrite se trouve aujourd'hui dans une situation délicate, alors que de nombreux titres font face à des situations financières précaires et que l'emploi journalistique connaît une période durable de repli en Amérique du Nord (Barthel, 2016). L'accélération de la transition numérique qui prolonge l'implantation d'outils numériques dans les rédactions de la presse écrite, amorcée de longue date (Ruellan et Thierry, 1998), conduit l'industrie de la presse à devoir composer avec une série de réaménagements importants. En ligne, la démultiplication des lieux de production, de diffusion et de consommation des contenus informationnels remettent sérieusement en question des modèles d'affaires établis autant que les stratégies de rétention du lectorat jusque là dominants dans le secteur de la presse écrite (Sonnac, 2009). On assiste ainsi depuis près d'une décennie à une décroissance marquée des revenus - publicitaires, abonnements ou petites annonces - pour les acteurs établis de l'information au profit de plateformes de diffusions natives du web qui ont très tôt fait valoir leur pertinence vis-à-vis des annonceurs (Bouquillion, 2008).

Ces questions se posent avec une acuité particulière dans de petits marchés linguistiques. En raison notamment de la faible taille du bassin de consommateurs, il est d'autant plus difficile de maintenir des revenus suffisants pour faire face à des coûts fixes de production qui restent eux sensiblement les mêmes que pour des marchés de plus grande taille (pour ces raisons, la production de contenus fait d'ailleurs rarement partie des activités poursuivies par les acteurs du numérique (Bouquillion, 2010)). Pourtant, au-delà de la seule dimension économique, parler de « petit marché », de « particularisme linguistique » ou de « société distincte » comme nous allons le faire ici, nous incite à prendre en compte les conséquences de ces transformations du secteur de la presse écrite dans un cadre beaucoup plus large. 
En effet, bien que notre objectif ici ne soit pas de problématiser la notion même de "petit marché ", il nous semble pertinent de préciser que les transformations qui affectent de manière spécifique le secteur de la presse écrite dans un tel contexte auront des retombées sur d'autres sphères - politique, sociale, culturelle - au sein desquelles ces industries s'insèrent, bien au-delà des seuls facteurs économiques, technologiques ou organisationnels. Nos propositions doivent donc nécessairement être prises dans ce cadre, où la portée des changements à l'œuvre dépasse celui d'une industrie en particulier.

Parmi les débats et les cas de figure proposés dans ce numéro pour les cas de petits marchés de la presse européens, cet article fait le point sur les spécificités de la transition numérique du secteur de l'information écrite au Québec, un petit marché francophone d'Amérique du Nord. Nous montrerons à ce sujet (1) que la transition numérique y a été plus lente et réalisée avec des moyens moins élevés qu'ailleurs en Amérique du Nord, mais également (2) que les groupes établis ont pris appui sur leurs expertises propres et leur positionnement éditorial antérieur pour baliser leur présence en ligne. Enfin, nous montrerons (3) en quoi la taille du marché et son relatif isolement culturel continuent à avoir une influence sur ces stratégies de déploiement et sur le maintien de la configuration du marché des médias. Dès lors, ramenée aux débats portant sur la rupture ou la continuité de pratiques professionnelles et organisationnelles en ligne, notre analyse tend à privilégier une voie médiane : celle de l'adaptation et de la transposition - non systématiquement à l'identique - de pratiques existantes. En ce sens, nous n'épouserons ni le discours de la « révolution » numérique ni celui de la reproduction intégrale de manières de faire existantes.

D'un point de vue méthodologique, les études de cas proposées ici s'appuient sur plusieurs terrains de recherche successifs et complémentaires, menés entre 2008 et aujourd'hui, portant sur différents aspects de la transition numérique pour les entreprises médiatiques québécoises, à savoir : (1) une analyse 
documentaire (rapports annuels des entreprises et revue de presse), une analyse des sites web et des entrevues avec des acteurs clé de la création d'une offre web pour les titres de presse francophone au Québec sur la période 1996-2009; (2) une analyse documentaire (conventions collectives et revue de presse) combinée à l'analyse des mécanismes de convergence dans les différents groupes médiatiques privés au Canada et une trentaine d'entrevues menées avec des journalistes de ces entreprises, réalisées dans le cadre d'une recherche dirigée par É. George au CRICIS entre 2008 et 2013 et (3) une veille informationnelle systématique portant sur la transition numérique du milieu médiatique et journalistique au Québec depuis le milieu des années 2000. Enfin, nous reprenons dans le cadre de l'analyse plusieurs rapports et données statistiques compilées par le Centre d'étude sur les médias à l'Université Laval.

\section{Québec, "société distincte » et marché médiatique de petite taille}

Pour comprendre les spécificités du marché de la presse au Québec, il est important de préciser brièvement le contexte social, culturel et politique dans lequel il se déploie : le Canada voit la coexistence de deux marchés médiatiques distincts qui correspondent à ses deux de ses nations constitutives ${ }^{2}$. Ici, les francophones, principalement établis au Québec (ils y représentent $80 \%$ de la population, alors que ceux-ci ne représentent que $22 \%$ de la population totale au Canada - Statistiques Canada, 2015), permettent à la province de jouir d'un statut particulier. Le Québec est également «nation culturelle » (Millette et Proulx, 2013), bâtie autour de la langue française, seule langue officielle commune depuis 1977. À ce titre, la province reste une société à part à l'échelle canadienne, et la principale enclave francophone en Amérique du Nord, dotée d'un marché médiatique comme d'institutions de soutien de la langue française et de la vie culturelle et même d'un vedettariat propre (Martin et al. 2010).

2 Le parlement canadien a reconnu en janvier 2006 que « les Québécois forment une nation au sein d'un Canada uni ». 
Existe, comme ailleurs, un lien particulier entre le développement et l'affirmation de l'identité québécoise et le déploiement d'un système médiatique local qui nourrit une culture médiatique forte. Par ailleurs, du point de vue du fonctionnement du marché médiatique, la question linguistique a son importance : alors que les médias canadiens anglophones doivent composer avec l'offre existant aux États-Unis, l'influence jouée par les autres médias francophones (européens notamment) semble nettement moins grande - les principales productions médiatiques consommées au Québec étant produites sur place (Martin et al. 2010).

Dans ce contexte, le marché de la presse écrite s'est développé de manière relativement isolée de celle du reste du Canada. Il représente aujourd'hui environ cinq millions de lecteurs francophones qui déclarent avoir un attachement plus marqué pour l'information et les médias, alors que la consommation médiatique d'information est plus élevée au Québec qu'ailleurs au Canada (Statistiques Canada, 2016). Parmi les modes de développement privilégiés au Québec et au Canada dans les industries de la culture et de la communication (ICC) au cours des quarante dernières années, on doit souligner l'accent mis sur la création de " champions nationaux » (George, 1998) qui ont diversifié leurs activités au sein des ICC - le développement de groupes dotés de monopoles sur des marchés régionaux et nationaux, capables de résister à la pression d'entreprises de grande taille, notamment ceux venant des États-Unis. Ce mécanisme, censé pallier la petitesse des marchés francophones à l'échelle canadienne et anglophone à l'échelle nord-américaine, a par ailleurs été doublé de mécanismes fiscaux visant à garantir que la propriété des médias resterait canadienne.

Ensemble, ces mécanismes ont structuré le marché autour d'une concentration de la propriété médiatique parmi la plus élevée à l'échelle occidentale, alors qu'un nombre toujours décroissant d'entreprises se partagent les secteurs de la culture et de la communication. Cette configuration particulière du marché, amorcée depuis la fin des années 1970, fait craindre 
périodiquement aux acteurs de la société civile un appauvrissement de la diversité des voix et du pluralisme de l'information (sur l'ensemble de ces enjeux voir, entre autres, l'ouvrage collectif dirigé par George et al., 2015). De fait, il reste extrêmement difficile pour de nouveaux entrants de s'imposer sur ce marché : aucun titre de presse pérenne n'a pu s'établir au Québec dans les trente dernières années, à l'exception de la création de deux titres gratuits à Montréal en 2001. Ceci est d'autant plus valable pour des structures indépendantes ne bénéficiant pas de l'aide de groupes établis, et ce même en tenant compte de l'explosion du numérique. Aujourd'hui, 14 titres quotidiens locaux (incluant les deux gratuits et le seul titre indépendant) sont diffusés au Québec, un nombre stable depuis le milieu des années 1980 (CEM, 2015a). Le marché est par ailleurs dominé par trois entreprises qui se partagent la quasi-totalité du lectorat francophone payant (elles n'étaient que deux avant que le groupe Gesca ne se départisse de ses titres régionaux en 2015). Un seul quotidien indépendant à la santé financière précaire, Le Devoir, subsiste sur le marché québécois.

Depuis la fin des années 1990, autant les revenus des entreprises que les tirages sont à la baisse, même s'il est impossible d'obtenir des données pour le Québec en particulier (la compilation a été effectuée par le CEM, 2015a). Ce déclin du lectorat restait néanmoins très progressif avant 2010, nettement moins marqué qu'ailleurs en Amérique du Nord. Par ailleurs, on a assisté, là aussi avec un temps de retard, à l'effondrement du marché publicitaire et du nombre d'exemplaires payants le premier étant plus marqué que l'autre depuis la crise financière de 2008-2009 : en fonction des données disponibles, les entreprises feraient face à une baisse de $25 \%$ des revenus publicitaires entre 2006 et 2011 au Québec (TVB, dans Brin et St-Pierre, 2013), alors qu'elle serait de plus 35\% sur la période 2000-2014 à l'échelle canadienne (CEM, 2015b). On se concentrera ici sur le cas de la presse quotidienne payante : le cas des hebdomadaires régionaux mériterait une contribution à elle seule, alors que ceux-ci sont plus durement touchés par la baisse des revenus, laissant craindre 
l'apparition de véritables déserts médiatiques et l'abandon progressif de tout investissement privé dans ce secteur.

\section{La transition numérique dans un marché hyperconcentré}

On a assisté au cours de la dernière décennie à une évolution des habitudes de lecture au Québec : le déclin de la presse écrite s'y fait de manière moins marquée qu'ailleurs alors qu'on même pu observer un maintien relatif des tirages entre 2009 et 2013 avant que cela ne reparte à la baisse. Principal facteur d'explication : une explosion du nombre d'exemplaires vendus à prix réduit ou distribués gratuitement par les entreprises (CEM, 2015a). Il est également envisageable d'invoquer la faiblesse du nombre de contenus de substitutions en ligne, en dehors des plateformes médiatiques déjà établies : la disponibilité de contenus en ligne ayant un intérêt pour le lectorat québécois reste moins diversifiée que pour des lecteurs anglophones ailleurs en Amérique du Nord.

De fait, l'offre québécoise professionnelle d'information sur internet peine à exister en dehors des grands groupes - autant du fait de la difficulté de générer des revenus de l'information journalistique que d'un manque de visibilité des productions (Carbasse, Demers et Fleury, 2016). Ainsi, contrairement aux nombreux espoirs qui avaient accompagné internet, le fonctionnement oligopolistique du marché des médias n'a pas disparu : les titres dominants hors ligne continuent à dominer en ligne (Reuters Institute for the Study of Journalism, 2016) alors que la plupart des médias natifs de l'internet ne sont toujours pas viables au Québec.

Il faut également souligner l'existence d'une rupture générationnelle sur la consommation de contenus numériques : alors que les moins de 35 ans affirment privilégier les contenus numériques, ce sont davantage vers la télévision et la presse que se tournent leurs aînés (Charlton et al., 2016). Ainsi, il n'est pas étonnant que la baisse de lecture des quotidiens papier soit nettement plus marquée chez les 18-24 ans, même si eux aussi 
conservent un attachement plus grand pour les plateformes traditionnelles que leurs homologues canadiens ou états-uniens (CEM, 2015a). De la même manière, la fréquentation des réseaux socionumériques est également plus marquée chez la jeune génération (CEFRIO, 2015).

La transition des acteurs de la presse sur internet s'est faite en plusieurs étapes depuis le milieu des années 1990, alors que le numérique a longtemps été cantonné à un rôle secondaire dans les activités de diffusion et de valorisation des groupes : l'intérêt des rédactions comme des directions ne s'est manifesté que très progressivement, bien que chaque entreprise ait eu une présence en ligne propre bien avant la fin de la décennie 1990 (Carbasse, 2009). En fait, les années 2008-2010 ont été des années charnières pour les entreprises qui ont alors cherché à systématiser et accroître la disponibilité des contenus sur leur site web, mais également à examiner les possibilités offertes par les applications numériques. L'un des principaux moteurs de cette accélération est sans nul doute la contraction du marché publicitaire de la presse québécoise depuis 2008 (Gagné, 2016). Cette transition est accompagnée de réduction de la taille de nombreuses rédactions (Brin et St-Pierre, 2013) : à quelques exceptions près (celle de La Presse notamment), on assiste au cours de la période à une baisse ou à une stagnation des effectifs rédactionnels, ceux de Québecor Média, principal groupe de la province, en tête. Là aussi, le déclin des effectifs connaît un sort nettement plus enviable qu'aux ÉtatsUnis et dans le reste du Canada qui voient une consolidation du marché, des pertes d'emplois et la fermeture de titres se succéder à un rythme beaucoup plus soutenu pendant la même période.

Ainsi, si les journalistes et les éditeurs ont été incités à s'approprier de manière plus efficace les plateformes numériques de diffusion - à commencer par leurs propres sites web et applications mobiles, ces transitions se sont faites à des moments différents pour chacun d'entre eux, en fonction des moyens propres disponibles, de la taille de chaque groupe, mais également de visions stratégiques distinctes au sujet du modèle économique 
qui doit permettre de pérenniser l'activité journalistique. Les expérimentations faites dans ce cadre tiennent à la fois compte de ce qui a pu être fait ailleurs, mais également du manque de moyens humains et de marges financières qui sont eux liées à la petitesse du marché, alors que les bénéfices réalisés au cours des décennies précédentes n'ont pas forcément été réinvestis dans l'outil de production. Dans ce contexte, cette transition numérique de la presse écrite québécoise se fait obligatoirement « sous contrainte » (Smyrnaios, 2013), à l'image des médias natifs de l'internet français étudiés par l'auteur, qui ne disposent ni d'une capitalisation importante, ni ne profitent des mécanismes de soutien étatique des titres déjà établis.

\section{Expérimentation et reproduction des modèles existants}

Alors que les modes diffusion de contenus en ligne sont restés relativement stables, les modèles d'affaires ont eux fait l'objet de plusieurs expérimentations depuis 2008. Avant cette date, les principaux groupes de presse avaient misé sur le modèle publicitaire, tandis que le quotidien indépendant Le Devoir, déjà moins dépendant des revenus publicitaires que ses concurrents, avait lui opté pour un modèle hybride, alliant contenus payants et contenus libres d'accès. Ainsi, il s'agit globalement d'une réplication des modèles et pratiques existantes, en tenant compte des manières de faire propres à chaque groupe de presse (Carbasse, 2009) : les entreprises ont des identités, un public, des traditions, des modes de gestion et des présences distinctes dans l'espace social, culturel et politique du Québec qui vont influencer leur approche de la publication sur internet. Par ailleurs, il importe de souligner le caractère nécessairement transitoire de la situation actuelle du marché des médias - au Québec comme ailleurs, alors que les modèles socioéconomiques existants s'essoufflent et que les groupes se trouvent dans une phase d'expérimentations. À l'heure actuelle, chaque entreprise a une stratégie propre : le groupe Québecor opte pour une diffusion multiplateforme gratuite, tandis que LaPresse a développé elle une application destinée aux tablettes. Le Devoir, lui, doit composer avec des 
moyens plus faibles et a choisi l'option d'un mur payant très tôt dans son histoire.

Journal de Montréal : du mur payant à la recherche du trafic maximal

Premier cas, celui du Journal de Montréal / Journal de Québec, deux tabloïds visant un lectorat populaire qui rassemblent $47 \%$ du tirage francophone en 2014 (CEM, 2015a). Ils sont adossés au groupe Québecor Média, principal acteur du secteur des médias, de la culture et des communications au Québec, incluant les activités de télécommunications. Les quotidiens n'ont créé leurs propres sites internet qu'en 2010, le groupe ayant fait auparavant le pari de faire converger l'intégralité de ses contenus écrits et audiovisuels sur le portail du groupe, canoe.ca (Carbasse, 2010). Les quotidiens ont notamment pu expérimenter avec les murs payants, mais également avec les lieux de diffusion en ligne alors que la rédaction montréalaise a connu une baisse de plus de $70 \%$ de ses effectifs suite à un très long lock-out décrété par l'employeur entre 2009 et 2011 (Brin et St-Pierre, 2013).

Pour les titres de ce conglomérat, la transition numérique - tant du point de vue des moyens mobilisés que des choix stratégiques - est tributaire de la variété des contenus et activités qui sont présents au sein du groupe (information, divertissement, communications). Celui-ci a revendiqué l'efficacité des mécanismes de convergence des activités de production et de diffusion, qui peut se faire au détriment de l'attention accordée à l'information, celle-ci étant désormais la branche la moins rentable des activités du conglomérat (Carbasse et Goyette-Côté, 2013; Anciaux, 2014). Les rapports annuels confirment ainsi que l'information et la production de contenus représentent une part décroissante des revenus dans les activités globales du groupe et que ce dernier concentre la majorité de ses efforts sur les activités de Vidéotron, sa filiale de télécommunications. En ce sens, pour les contenus, on observe même un recentrement autour des marques les plus fortes, alors que le groupe s'est départi de 
ses publications de quotidiens au Canada anglais en 2014 pour ne conserver que ses titres francophones. En effet, alors que les propriétés anglophones n'étaient adossées à aucune activité de télécommunication au Canada anglais, elles devenaient dès lors moins stratégiques pour Québecor, dont les activités de fournisseur d'accès internet, de téléphonie mobile et de câblodistribution sont elles centrées au Québec et lui permettent de créer de véritables synergies avec les activités de contenus francophones, comme de réaffirmer son ancrage local sur le territoire québécois.

La stratégie de convergence particulièrement aboutie mise en place par le groupe, conjuguée aux déficits des activités de contenus a eu des impacts du point de vue de l'emploi : la rédaction du Journal de Montréal est ainsi passée de 133 à 34 travailleurs entre 2009 et 2012 (Brin et St-Pierre, 2013), alors que les décisions de déploiement numérique sont désormais prises en amont des journalistes, dans la division Québecor numérique. Dans ce cadre, Quebecor avait misé originellement sur la création d'une agence de presse internet - QMI - pour réaliser une partie des activités journalistiques en produisant des contenus pour l'ensemble des titres du groupe. Celle-ci a néanmoins vu ses effectifs décroître assez rapidement, notamment en raison du recentrement sur une offre journalistique en français. Sur les sites des journaux, l'offre de contenus reprend les modes de présentations privilégiés des tabloïds : très forte identité visuelle axée sur la photographie et des articles courts. Comme pour les quotidiens papier, les sites du groupe bénéficient d'un lectorat important - plus de $3 \mathrm{M}$ de visiteurs uniques par mois, 1,8M sur mobile (Turbide, 2016).

Alors que le groupe avait tenté le pari du mur payant en 2013 (l'accès à tous les articles coûtait $6,95 \$$ par mois), celuici a été abandonné en janvier 2016. L'entreprise vise désormais la visibilité maximale de ses contenus en ligne et souhaite aller de l'avant avec une publication sur les réseaux socionumériques, Facebook en tête, qui représentent désormais la majorité de ses visiteurs entrants. D'ailleurs, le retour de la gratuité a coïncidé avec l'embauche de publicitaires pour développer une section 
« sac de chips », des nouvelles insolites à fort potentiel de clics (clickbait), une stratégie proche de celle mobilisée entre autres par le site Buzzfeed (Cipriani, 2016). Les rédactions ont également été les premières à faire le saut dans les articles instantanés sur la plateforme Facebook au Québec dès la fin de 2015 (Journal de Montréal, 2015).

Avec ses marques d'information, Québecor vise désormais à recréer en ligne la formule qui a fait son succès sur la presse papier : une information gratuite visant une audience maximale et disponible par toute une panoplie de canaux de diffusion. Toutefois, le groupe s'inspire également des pratiques commerciales les plus efficaces des médias natifs d'internet connaissant du succès. L'avenir immédiat de ces titres ne semble pas menacé : le Journal de Montréal et le Journal de Québec conservent une dimension symbolique forte et sont des marques phares de la production de contenus d'information pour le groupe, qui en utilise les contenus pour promouvoir ses activités rentables (téléphonie, internet et câblodistribution).

\section{Investir dans l'avenir : le cas de la Presse+}

Plus connu à l'échelle internationale, le cas de La Presse+, disponible depuis 2013, représente un produit particulièrement innovant du point de vue journalistique et témoigne d'une tout autre vision du modèle d'affaires, axée sur la mise en valeur d'une application destinée aux tablettes et la création d'un rendezvous quotidien. Pour aboutir à ce résultat, l'entreprise éditrice de La Presse, Gesca, filiale de la multinationale financière Power Corporation, a pu bénéficier d'un investissement conséquent réalisé par les actionnaires. Les propriétaires ont de plus fait un pari audacieux et symbolique, celui de l'abandon de l'édition papier en semaine depuis le $1^{\text {er }}$ janvier 2016, pour ne livrer les contenus que sur l'application (Crevier, 2015).

Le quotidien montréalais La Presse avait une présence en ligne déjà établie : premier quotidien à mobiliser dès 2008 
l'intégralité de sa rédaction pour le web qui devenait la première instance de publication, le quotidien papier n'arrivant qu'ensuite (Carbasse, 2009), l'entreprise a poursuivi sa stratégie numérique avec le développement d'une application créée à partir de zéro, initialement connue comme "Plan iPad", qui permet la visualisation de contenus journalistiques multimédia, organisés par « écrans » au sein desquels il est possible de naviguer et qu'il est possible de déployer pour de l'information complémentaire. Elle aura nécessité un investissement total de 40M\$ entre 2011 et 2013, ainsi que la mobilisation de 70 employés dédiés à son développement. Lancée en 2013 sur les tablettes iPad, elle est devenue disponible pour l'environnement Android l'année suivante.

Avec La Presse+, le groupe reste sur un modèle économique basé sur la publicité adaptée au format tablette et des sections dédiées au marketing de contenu via la section "Xtra », mise en place en dépit des réticences de la rédaction et largement remarquée par la profession. Le groupe a d'ailleurs été condamné à ce sujet par le Conseil de presse du Québec en 2016, notamment en raison d'une identification suffisante du caractère commandité de ce type de contenus (Baillargeon, 2016a). Le choix de la mise à disposition gratuite des contenus est revendiqué ouvertement par la direction depuis le lancement de l'application, les dirigeants estimant qu'il leur est impossible de faire payer de l'information au Québec. La principale raison évoquée tient à la réticence de la jeune génération, leur public cible, à payer pour de l'information, alors qu'ils souhaitent créer de nouvelles habitudes de lecture. En ce sens, la stratégie est cohérente avec celle qui avait été mise en place pour le portail cyberpresse.ca (devenu lapresse.ca depuis) où l'intégralité des contenus était mise gratuitement à disposition du lecteur. Autre source de revenus escomptée par le groupe : la vente de l'application à d'autres entreprises de presse. En juin 2016, le Toronto Star est le seul client de Gesca pour ce secteur, alors que le quotidien torontois a lancé le Star Touch en 2015 sans pour autant remporter le même succès que celui de l'application de Gesca. 
Néanmoins, il reste impossible à l'heure actuelle de savoir si le modèle choisi est viable. S'il s'agit effectivement d'un pari audacieux et d'un produit unique, aucune donnée financière n'est dévoilée à son sujet, dans la poursuite de la politique de Power Corporation de ne pas détailler la situation financière de ses filiales depuis le début des années 2000. Pour l'instant, il faut donc s'en remettre aux assurances données par les propriétaires : le discours autopromotionnel n'aborde la question qu'en termes d'abonnés et de portion prise par l'application dans les revenus publicitaires globaux (eux aussi non divulgués) du groupe. Ainsi, Gesca souligne que désormais $85 \%$ de ses revenus sont générés par le numérique, sans pour autant indiquer l'évolution de ces derniers (Roulot-Ganzmann, 2016). De son côté, Roy (2015) souligne ainsi que, non seulement l'application reste potentiellement déficitaire, mais que l'opacité qui règne sur les résultats des activités médias du groupe ne permet pas d'évaluer la viabilité financière des activités indépendamment des garanties données par la direction de l'entreprise. Au cours de l'année 2016, plusieurs cadres du groupe ont annoncé publiquement viser l'équilibre budgétaire pour les activités de la rédaction. Ainsi, alors que la période de développement de l'application avait permis d'embaucher un grand nombre d'informaticiens et de journalistes, l'abandon de l'édition papier a coïncidé avec une série de licenciements, pour ramener le nombre d'employés à 660 (Crevier, 2015). Il s'agit néanmoins d'une légère augmentation des effectifs rédactionnels entre 2011 et 2016.

Quoi qu'il en soit, sur le court terme La Presse+ reste indéniablement un succès de lecture : en deux ans, elle a réussi à rassembler 240000 utilisateurs par jour, alors que, chaque semaine, 580000 utilisateurs téléchargent et ouvrent une édition sur leur tablette, des chiffres qui dépassent largement le tirage du quotidien. À titre de comparaison, La Presse était diffusée auprès de 81000 abonnés payants en semaine au moment de mettre fin à l'édition papier et 30000 lecteurs supplémentaires pour l'édition du samedi, qui elle n'a pas été supprimée. Il s'agissait du même niveau qu'au début du 20e siècle (Baillargeon, 2016b). 
Selon les mêmes sources, les usages de lecture sont également marqués d'un point de vue générationnel alors que les 25-54 ans représentent $63 \%$ du lectorat. Toujours selon les données divulguées par Gesca, les lecteurs restent en moyenne 40 minutes sur leur édition. Ainsi, la mobilisation d'une audience nettement plus attentive que sur le papier peut en partie justifier la transition vers le « tout numérique » et l'abandon du papier en semaine : les revenus générés auprès des annonceurs par le lectorat particulier de l'application tablette permettent vraisemblablement de compenser la perte des revenus publicitaires et des revenus de vente du quotidien papier.

Avec l'arrêt de la publication papier au $1^{\text {er }}$ janvier 2016, on a pu par contre noter une augmentation des abonnements chez ses concurrents, à hauteur notamment de $15 \%$ pour Le Devoir qui indique profiter également des fruits de son travail de repositionnement entamé de plus longue date. Si cela doit se confirmer avec le temps, cela nous incite à penser que la stratégie $\mathrm{du}$ 《 tout-tablette » rencontre des limites, notamment en termes d'habitudes de lecture, mais également en termes d'équipement des ménages, alors que les cellulaires n'ont pas accès à l'application.

Enfin, et c'est particulièrement notable du point de vue de la réorganisation du marché de la presse au Québec, le pari de la tablette pour La Presse s'est doublé d'un repositionnement stratégique pour l'entreprise qui s'est départie de ses six quotidiens régionaux à un groupe détenu par un proche du Parti Libéral du Canada, sans que le montant de la vente ou l'origine des capitaux soit connue (Bergeron, 2015). Cette vente des activités médiatiques régionales au groupe Capitale Médias en 2015 concrétise le manque d'investissements réalisés par Gesca sur ses autres titres : ceux-ci accusaient alors un retard historique sur le web, alors que leurs contenus étaient diffusés (et continuent à l'être en 2016) sur lapresse.ca et ne disposaient d'aucune application mobile propre. Depuis la transaction, les titres de Capitale Médias se sont dotés d'applications mobiles pour chacun des titres et ont amorcé des partenariats multimédias 
avec des stations de télévision en région (Buchinger, 2015), au prix de plusieurs concessions des rédactions, incluant la mise en place d'un plan de départs volontaires.

Indépendance, précarité et mur payant : la place à part du Devoir

À une autre échelle, Le Devoir, quotidien centenaire et indépendant qui représente environ $3 \%$ du tirage payant au Québec, doit poursuivre une transition numérique à la hauteur de ses moyens. S'il a connu des résultats financiers positifs durant la décennie 2000, les difficultés financières sont presque constitutives de son identité (Coulombe et Charron, 2012). La transition s'y fait véritablement sous contraintes, alors que les liquidités et les moyens humains dont dispose le journal, dont l'information est le seul pôle d'activité, sont sans commune mesure avec ceux de ses concurrents adossés respectivement à un groupe de communication et à une multinationale de la finance et de l'assurance.

Dès le début des années 2000, le quotidien a choisi de faire payer pour une partie de ses contenus, capitalisant sur un rapport particulier à son lectorat existant de longue date. En effet, contrairement à ses concurrents, Le Devoir maintient un ratio globalement égal entre revenus d'abonnements et revenus publicitaires. Dans sa mouture de 2001, le site web du Devoir réserve donc un tiers des contenus à ses abonnés et offre un abonnement numérique qui s'avère rentable dès le départ (Carbasse, 2009). Ce modèle freemium sera remplacé en 2015 par un mur payant qui autorise la consultation de 15 articles gratuitement par mois (FPJQ, 2015).

Paradoxalement, durant la décennie 2000, son activité web fut la plus rentable des cas étudiés dans cet article. Faire payer pour des contenus déjà amortis par les abonnements papier en faisait un cas unique. Toutefois, au cours des dernières années, le journal cumule des pertes importantes qui contrastent avec près d'une décennie de résultats financiers excédentaires, pertes 
liées principalement à l'effondrement des revenus publicitaires, une situation qui restreint d'autant la marge de manœuvre pour le déploiement de nouvelles activités.

Pour faire face à celle-ci et continuer à déployer ses activités en ligne, l'entreprise mise à nouveau sur le lien fort qu'elle entretient avec son lectorat : outre une campagne d'appel aux abonnements et aux dons, la direction a mis en place une stratégie de sociofinancement autour des «Amis du Devoir », cherchant à mobiliser un groupe de 200 donateurs qui s'engageraient à contribuer à hauteur de $1000 \$$ chacun sur plusieurs années, les sommes dégagées devant aller aux activités de développement du journal (Descôteaux, 2015). Le journal se trouve d'ailleurs dans une période de transition alors que la direction et rédaction en chef ont changé en 2016, suivis par une série d'embauches et de création de postes à des secteurs stratégiques du numérique.

\section{Une transition numérique sous contraintes : entre continuités de l'offre et recherche de nouvelles sources de revenus}

Le relatif isolement culturel et médiatique dont a joui, avec d'autres, le secteur de la presse au Québec est en somme une arme à double tranchant. Ce qui a historiquement été une protection vis-à-vis de l'arrivée de nouveaux entrants sur des marchés oligopolistiques et une garantie de résultats économiques confortables pour les grands groupes établis, limite aujourd'hui les moyens dont les mêmes acteurs disposent pour assurer leur transition numérique et subsister en espérant trouver un modèle économique viable. Alors qu'ils se sont trouvés en position d'attente face à internet, notamment en raison du manque d'incitatifs d'augmenter leur présence en ligne avant la crise financière de 2008, les protagonistes de la presse écrite québécoise cherchent aujourd'hui un modèle d'affaires performant dans une situation de contraintes. Ceci ne veut évidemment pas dire que la presse québécoise n'a pas tenté de s'approprier internet avant la crise financière : tous les titres ont, d'une manière ou d'une autre, 
commencé à occuper le terrain sur ce nouveau canal de diffusion. Quelques éléments d'analyse s'imposent à ce sujet.

Tout d'abord, loin des discours visant à faire équivalent « numérique » et « rupture », la transition des entreprises de presse sur le web dans le contexte québécois est plutôt marquée par une certaine continuité dans les pratiques à la fois de production et de valorisation des contenus informationnels, la principale variable d'ajustements se trouvant dans l'évolution des stratégies de diffusion. Cette continuité doit nécessairement inviter l'observateur à penser les pratiques dans la durée et dans une logique de déstructuration-restructuration (Demers, 2007) des stratégies entrepreneuriales d'une ampleur moins importante que celle envisagée au début du processus. Dans les cas évoqués ici, les stratégies de diffusion pour le web évoluent dans une logique d'adaptation progressive et incrémentale, à mesure que de nouveaux canaux - la plupart indépendants des entreprises productrices de contenus elles-mêmes - rassemblent une masse de lecteurs. La transition numérique - qu'il s'agisse d'un marché de petite taille ou non - doit donc nécessairement être observée dans la durée, alors que les routines de production et de diffusion auront pris plus d'une décennie pour adapter leur rythme et leurs pratiques professionnelles aux particularités d'une offre journalistique centrée sur le web. Qui plus est, l'existence d'un marché linguistique et culturel unique en Amérique du Nord, où la proximité cultivée entre les marques de presse établies et le lectorat, permet de comprendre la lenteur et la magnitude du déploiement d'activités en ligne par les acteurs observés.

Penser la restructuration de la presse québécoise en ligne comme une réplication d'un certain nombre de modes opératoires au niveau de l'offre sur de nouvelles plateformes de diffusion, implique de s'attarder à la transposition des modèles d'affaires qui avaient dominé hors ligne pour chacun des acteurs. Ainsi, alors que le mur payant est désormais la norme sur le marché des États-Unis pour les quotidiens ayant une forte circulation (Williams, 2016), on constate un échec des principales tentatives 
d'implanter ce modèle sur le marché québécois - et canadien - et une domination nette de la stratégie visant à mettre à disposition gratuitementl'information par les principaux groupes médiatiques, lorsque le financement par les abonnements était déjà minoritaire face aux revenus issus de la publicité. À l'inverse, le seul acteur ayant historiquement fait le pari d'un soutien direct de la part de ses lecteurs, Le Devoir, s'est lui naturellement tourné vers une forme de mur payant, rentable, dès le début des années 2000 et n'y a pas renoncé depuis. Pour les premiers acteurs, il est possible de penser que l'absence d'une masse critique de consommateurs payants pour l'information québécoise rende extrêmement difficile de limiter un tant soit peu le nombre de pages vues - et donc les revenus publicitaires qui y sont associés - pour un éditeur. L'isolement culturel et le caractère distinct de la consommation médiatique et culturelle ne garantissent toutefois pas que les consommateurs d'information aient une plus grosse propension à payer. Dans un contexte de petit marché médiatique, la capacité à rejoindre le plus grand nombre de lecteurs locaux peut être vu comme un argument décisif vis-à-vis des annonceurs lorsque vient le temps des décisions d'achat de placement publicitaire. En revanche, cette étroitesse du marché pose également la question du cas de figure inverse : alors que le marché des annonceurs comme celui des lecteurs reste partiellement captif, ne faire reposer le modèle de valorisation de l'information que sur d'hypothétiques revenus publicitaires implique également une certaine prise de risque de la part des entreprises qui ne sont aucunement assurée de l'atteinte de revenus suffisants. À l'image du Devoir qui est à l'heure actuelle le seul titre qui tente de combiner abonnements payants et revenus publicitaires, l'ensemble des entreprises doit donc encore trouver la meilleure manière de diversifier leurs sources de revenus alors que, pour l'instant, les activités journalistiques ne semblent pas être viables par elles-mêmes.

Dans le même ordre d'idées, l'étroitesse du marché médiatique en contexte «protégé » a eu des impacts importants sur l'organisation du marché des médias et sur les liens entretenus entre les activités journalistiques et d'autres secteurs industriels, 
lesquels se poursuivent en contexte numérique. La petitesse du marché a ainsi pu précipiter des mouvements de financiarisation et de concentration de la propriété médiatique comme des réorganisations industrielles entre industries de la culture et de l'information d'une part et industries de la communication d'autre part, décrites par Bouquillion (2008). Les politiques de propriété canadienne des médias et l'inaction des pouvoirs publics en matière de concentration de la propriété de la presse au Québec et au Canada (Tremblay, 2015) ont donc mené beaucoup plus tôt qu'ailleursà la constitution de grands groupes de la communication, de la culture et de l'information, capables de déployer des moyens nécessaires à la poursuite des activités de production de contenus tout en la rationalisant par vagues successives. Bien avant que ces mouvements ne soient observables dans d'autres marchés de plus grande envergure - marché français des médias entre autres - ces rapprochements ont diversifié les régimes de propriété des groupes de presse avec l'apparition de modes de gestion managériaux. Ceux-ci ont pu à leur tour, en conjugaison avec une série d'autres facteurs, avoir une influence sur les moyens humains et financiers alloués à l'information, ainsi que sur les finalités de la transition numérique des différents groupes : force est de constater que pour Gesca, à la fois le mode de gestion largement influencé par la famille actionnaire de contrôle et le fait que l'information soit une activité historiquement extrêmement minoritaire dans les résultats financiers de Power Corporation, ont permis de mobiliser les sommes et ressources humaines nécessaires à la création de $\mathrm{La}$ Presse +. À l'inverse, la recherche de synergies entre les activités du groupe Québecor s'est traduite par une rationalisation accrue des activités d'information, moins rentables que d'autres secteurs d'activités connexes. Dans tous les cas, on a visé de longue date la recherche de ressources supplémentaires pour subventionner en partie des activités d'information « dure », jugées économiquement moins rentables que des contenus plus proches du journalisme de consommation ou divertissement. Cette diversité des stratégies, des finalités et des moyens accordés à la production de contenus ayant une teneur sociale, politique ou culturelle particulière devra 
nécessairement être suivie de près dans un contexte d'incertitudes sur la viabilité des modèles socioéconomiques retenus.

Enfin, le caractère particulier du « produit journalistique », subventionné en partie par des activités d'autres secteurs industriels, pose la question des mécanismes qui peuvent s'y substituer dans l'éventualité où la contraction des revenus publicitaires ou des abonnements payants serait amenée à se confirmer. En l'absence d'une culture du mécénat forte comme celle du Canada anglais ou des États-Unis, la question de l'intervention de l'État se pose désormais au Québec. Longtemps refusé par les journalistes comme les éditeurs au nom du respect de leur indépendance, le soutien financier ou logistique de l'état aux médias d'information a connu un regain d'intérêt de la part des éditeurs et des journalistes en 2016. Si les propositions précédentes faites en ce sens concernaient davantage le soutien aux médias audiovisuels (par le biais des diffuseurs publics Radio-Canada et Télé-Québec) ou communautaires, celui de la presse semble aujourd'hui être l'objet de beaucoup d'attentions: autant des acteurs de la société civile que des syndicats de journalistes et des éditeurs de presse ont appelé à la mise en place de mécanismes de soutien temporaires du gouvernement québécois, destiné à leur permettre de finaliser leur transition sur internet (Gagné, 2016). De son côté, le gouvernement fédéral a amorcé un chantier d'examen de ses mécanismes de soutien et de valorisation des industries de la culture et de la communication et s'interroge sur la pertinence de soutenir les acteurs régionaux de l'information.

\section{Références}

Anciaux, A. (2014). Entre discours et transformations des modèles d'affaires, la place nouvelle du médiatique et du journalisme dans un groupe industriel de communication canadien. Dans F. Le Cam \& D. Ruellan (Éd.), Changements et permanences du journalisme. Paris : L'Harmattan, 41-59.

Baillargeon, S. (2016a). Le masque démasqué. Le Devoir, 30 janvier. Disponible à : http://www.ledevoir.com/societe/medias/461651/le-masque-demasque 
Baillargeon, S. (2016b). La tablette moins. Le Devoir, 21 mars. Disponible à : http:// www.ledevoir.com/societe/medias/466040/medias-la-tablette-moins

Barthel, M. (2016). Newspapers: Fact Sheet. State of the News Media 2016 - Pew Research Center. Disponible à : http://www.journalism.org/2016/06/15/newspapersfact-sheet/

Bergeron, M. (2015). Les journaux régionaux de Gesca sont vendus. La Presse, 18 mars. Disponible à: http://affaires.lapresse.ca/economie/medias-ettelecoms/201503/18/01-4853323-les-journaux-regionaux-de-gesca-sont-vendus. php

Bouquillion, P. (2008). Les industries de la culture et de la communication : les stratégies du capitalisme. Grenoble: PUG.

Bouquillion, P. (2010). Les industries de la culture face aux industries de la communication: l'actualité des théories des industries culturelles. Observatoire des mutations des industries culturelles, série "Perspectives transerversales ». Disponible à : http://www.observatoire-omic.org/pdf/Bouquillion_industries_ culture_communication_theorie_industries_culturelles.pdf

Brin, C., \& M. St-Pierre. (2013). Crise des médias et effectifs rédactionnels au Québec. Rapport de recherche destiné au Centre d'étude sur les médias. Disponible à : http:// www.cem.ulaval.ca/pdf/Crise $\% 20 \mathrm{des} \% 20$ medias $\% 20$ et $\% 20$ effectifs.pdf

Buchinger, J. (2015). Nouvelles applications pour les quotidiens de Groupe Capitales Média. Infopresse, 14 octobre. Disponible à : http://www.infopresse.com/ article/2015/10/14/nouvelles-apps-pour-les-quotidiens-de-groupe-capitales-medias

Carbasse, R. (2009). Les stratégies des entreprises de presse quotidienne francophone québécoises dans le déploiement d'activités en ligne : 1996-2009 Mémoire. Montréal (Québec, Canada), Université du Québec à Montréal, Maîtrise en communication. 111p. Disponible à : http://www.archipel.uqam.ca/2497/1/M11165.pdf

Carbasse, R., Demers, F. \& Fleury, J.-M. (2016). La critique des médias au Québec francophone par les citoyens-entrepreneurs de nouveaux médias, de nouvelles pratiques et de nouveaux contenus. Quelques cas. Colloque "Les Médias face à leurs publics », Université d'Ottawa, 19 mai 2016.

Carbasse, R., \& Goyette-Côté, M.-O. (2013). L'impact des stratégies de convergence sur le travail journalistique : le cas des travailleurs de l'information de Québecor Média. Recherches en Communication, 39, 13-29.

Claus, S. (2015). Concentration et domination des médias au Québec, un état des lieux. Dans George, É. et al. (Éd.) Concentration de la propriété des médias, changements technologiques et pluralisme de l'information. Québec : PUL. 37-58

CEFRIO (2015). Les médias sociaux : une plus forte présence dans le processus d'achat des québécois. NETendances, 6-1. Disponible à : http://www.cefrio.qc.ca/ media/uploader/FasciculeNETendances2015-MdiasSociaux-Versionfinale.pdf

Centre d'études sur les médias (2015a). La presse quotidienne. Portraits sectoriels $d u$ CEM. Disponible à : http://www.cem.ulaval.ca/pdf/pressequotidienne.pdf

Centre d'études sur les médias (2015b). Données financières. CEM. Disponible à : http://www.cem.ulaval.ca/pdf/Donneesfinancieres.pdf

Charlton, S., Giroux, D., \& Lemieux, M. (2016). Les Québécois et l'information à l'ère numérique. Rapport du CEM. Disponible à : http://www.cem.ulaval.ca/pdf/ Quebecnumerique.pdf 
Cipriani, J.-P. (2016). Médias locaux vs. Géants mondiaux. Voir, 19 février. Disponible à : https://voir.ca/societe/2016/02/19/dossier-special-medias-locaux-vs-geantsmondiaux/

Coulombe, D., \& Charron, J. (2012). L'évolution de la situation financière du Devoir à la lumière de ses états financiers, Communication, 29(2), DOI : 10.4000/ communication. 2798

Crevier, G. (2015). La Presse papier sera remplacée par La Presse+ du lundi au vendredi dès le $1^{\text {er }}$ janvier. La Presse, 16 septembre. Disponible à : http://www.lapresse.ca/ debats/mot-de-lediteur/201509/16/01-4901051-la-presse-papier-sera-remplaceepar-la-presse-du-lundi-au-vendredi-des-le-1er-janvier.php

Demers, F. (2007). Déstructuration et restructuration du journalisme. Tic\&Société, 1(1), $28-55$.

Descoteaux, B. (2015). Nouveau microsite pour appuyer Le Devoir. Le Devoir, 26 mars. Disponible à : http://www.ledevoir.com/societe/medias/435546/nouveaumicrosite-pour-appuyer-le-devoir

FPJQ (2015). Le Devoir instaure un « mur payant» pour les abonnés. FPJQ. Disponible à : http://www.fpjq.org/le-devoir-instaure-un-mur-payant-pour-les-non-abonnes/

Gagné, J.-P. (2016). Quest-ce qu'on attend pour venir au chevet des médias écrits? Les Affaires, 11 juin. Disponible à : http://www.lesaffaires.com/secteurs-d-activite/ general/qu-attend-on-pour-venir-au-chevet-des-medias-ecrits-/588021

George, É. (1998). Service public de la télévision et marchandisation de la communication. Sciences de la société, 43, 147-163.

George, É. (Éd.) (2015), avec la collaboration de Brunelle, A.-M. \& Carbasse, R. Concentration de la propriété des médias, changements technologiques et pluralisme de l'information. Québec: PUL.

Goyette-Côté, M.-O., Carbasse, R., \& É. George (2012). Converging Journalism. Journalism Studies, 13(5-6), 753-762.

Journal de Montréal (2015). Des articles instantanés - le Journal, premier média francophone invité par Facebook. Journal de Montréal, 16 décembre. Disponible à : http://www.journaldemontreal.com/2015/12/16/des-articles-instantanes

Martin, C., de la Durantaye, M., Lemieux, J., Baillargeon, J-P., \& Pronovost, G. (2010). Le modèle québécois des industries culturelles. Rapport de recherche de l'action concertée "Le développement des entreprises et des organismes dans le secteur culturel ", avril. Disponible à : http://www.stat.gouv.qc.ca/statistiques/culture/ modele-qc-industries.pdf

Millette, J., \& Proulx, S. (2013). Médias et transnationalité. Le rôle des médias et d'Internet dans la trajectoire identitaire de jeunes (18-25 ans) issus de l'immigration. Rapport du CEM. Disponible à : http://www.cem.ulaval.ca/pdf/ MediasTransnationalite.pdf

Reuters Institute for the Study of Journalism, (2016). Digital News Report 2016. Disponible à : http://www.digitalnewsreport.org/

Roulot-Ganzman, H. (2016). Chacun cherche (encore) son modèle d'affaires. Projet $J$. Disponible à : http://projetj.ca/2016/02/10/chacun-cherche-encore-son-modeledaffaires/

Roy, J.-H. (2015) La valeur de l'expérience de La Presse+. Jhroy.ca. Disponible à : http://jhroy.ca/2015/12/fin-de-la-presse-papier/

Ruellan, D., \& Thierry, D. (1998). Informatisation et identités journalistiques en presse locale. Les Cahiers Du Journalisme, 5, 104-109. 
Sonnac, N. (2009). L'économie de la presse : vers un nouveau modèle d'affaires. Les Cahiers Du Journalisme, 20, 22-43.

Smyrnaios, N. (2013). Les pure players entre innovation journalistique et contrainte économique : les cas de Rue 89, Owni et Arrêt sur images. Recherches en communication, 39, 133-150

Statistiques Canada, (2015). Effectif et proportion de la population ayant déclaré le français selon la caractéristique linguistique, Québec, 2006 et 2011. Statistiques Canada. Disponible à : https://www12.statcan.gc.ca/census-recensement/2011/assa/98-314-x/2011003/tbl/tbl3_1-2-fra.cfm

Statistiques Canada, (2016). L'utilisation des médias pour suivre les nouvelles et l'actualité. Résultats de l'Enquête sociale générale, Statistiques Canada. Disponible à : http://www.statcan.gc.ca/pub/89-652-x/89-652-x2016001-fra.htm

Tremblay, G. (2015). Concentration de la propriété et pluralisme de l'information, un débat suranné? Dans George, É. et al. (Éd.) Concentration de la propriété des médias, changements technologiques et pluralisme de l'information. Québec : PUL. 19-36

Turbide, M (2016). Merci à nos 3,1 millions de lecteurs sur le web. Journal de Montréal, 12 mars. Disponible à : http://www.journaldemontreal.com/2016/03/11/merci-anos-31-millions-de-lecteurs-sur-le-web

Williams, A. (2016). Paying for Digital News: The Rapid Adoption and Current Landscape of Digital Subscriptions at U.S. newspapers. American Press Institute. Disponible à : https://www.americanpressinstitute.org/publications/reports/digitalsubscriptions

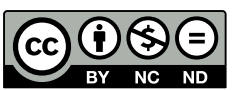

Publié sous la licence Creative Commons «Attribution - Pas d'Utilisation Commerciale - Pas de Modification 4.0 International» (CC BY-NC-ND) 